\title{
Analysis of modular organisation of interaction networks based on asymptotic dynamics ${ }^{\star}$
}

\author{
Franck Delaplace ${ }^{1, \star \star}$, Hanna Klaudel ${ }^{1}$, \\ Tarek Melliti ${ }^{1}$, and Sylvain Sené ${ }^{1,2}$ \\ 1 Université d'Evry - Val d'Essonne, IBISC, EA 4526, 91000 Evry, France \\ 2 Institut rhône-alpin des systèmes complexes, IXXI, 69007 Lyon, France
}

\begin{abstract}
This paper investigates questions related to modularity in biological interaction networks. We develop a discrete theoretical framework based on the analysis of the asymptotic dynamics of biological interaction networks. More precisely, we exhibit formal conditions under which agents of interaction networks can be grouped into modules, forming a modular organisation. Our main result is that the conventional decomposition into strongly connected components fulfills the formal conditions of being a modular organisation. We also propose a modular and incremental algorithm for an efficient equilibria computation. Furthermore, we point out that our framework enables a finer analysis providing a decomposition in elementary modules, possibly smaller than strongly connected components.
\end{abstract}

Keywords: modularity, interaction networks, discrete dynamics, equilibria.

\section{Introduction}

The analysis of the relations between the structure of a biological system and the related biological functions that identify specific states describing particular behaviours is among the most challenging problems [1] at the frontier of theoretical computer science and biology. Let us introduce an illustration of these structure/function relations. On the one hand, gene regulation may be structured into a directed graph, called the interaction graph, from which a dynamics is computed. On the other hand, the attractors (i.e., stable configurations and/or sustained oscillations) of such a dynamics identify the functions of the system. For instance, for the bacteriophage $\lambda$, the reciprocal regulations between genes Cro and $\mathrm{cI}$ induce two biological functions, namely the lysis and the lysogeny $[2,3,4]$, each corresponding to a distinct attractor.

\footnotetext{
* This work is supported by the project SYNBIOTIC of French National Agency for Research, ANR BLAN-0307-01.

${ }^{\star \star}$ Corresponding author: franck.delaplace@ibisc.univ-evry.fr.
} 
Generally, studying complex biological functions relies on their decomposition into sub-functions identifying some basic behaviours. Each subfunction is supported by a part of the structure. In the context of gene regulation, this part corresponds to a sub-graph of the interaction graph. Thus, the whole system can be viewed in a modular way, where modularity establishes the link between the parts and their related sub-functions. The module composition refers to a structural composition as well as a dynamical one.

In the literature, methods related to module discovery in interaction networks are generally based on both the analysis of the network structures (a field close to graph theory) and the study of their associated dynamics [5]. Structural analysis identifies sub-networks with specific topological properties motivated either by a correspondence between topology and functionality $[6,7]$ or by the existence of statistical biases with respect to random networks [8]. Specific topologies like cliques [9], or more generally strongly connected components (SCCs) are commonly used to reveal modules by structural analysis. Particular motifs $[10,11]$ may also be interpreted as modules viewed as basic components. They represent over-represented biological sub-networks with respect to random ones. Moreover, dynamical analysis lays on the hypothesis that expression profiles provide insights on the relations between regulators, modules being possibly revealed from correlations between the expressions of biological agents. For instance, using yeast gene expression data, the authors of $[12,13]$ inferred modules from co-regulated genes and the condition under which the regulation occurs. As a consequence, the discovery of a modular organisation in biological interaction networks is closely related to the influence of agents on one another and needs to investigate their expression dynamics $[14,15]$.

In $[16,17,18]$, the authors point out the need to relate structure and function to deal with modular organisations. The objective of this article is to define formally the notion of modular organisation as a list of modules together with a composition operation so that the dynamics of the module composition meets the global dynamics of the system. Indeed, modularity is somehow related to an invariance property of module asymptotic dynamics against regulatory perturbations of other modules [19], which supports the idea of viewing the global dynamics as the composition of the module's dynamics. Thus, using a discrete model of biological interaction networks [20,21], we propose an approach that analyses the conditions of module formation and characterises the relations between the global behaviour of a network and the local behaviours of its components. We 
show under which conditions interaction networks can be divided into modules. As main results, we propose a modular and incremental algorithm to compute equilibria and we show that the conventional structural decomposition into strongly connected components fulfils the formal conditions of being a modular organisation. Furthermore, we show that our framework enables a finer analysis providing a decomposition in elementary modules, possibly smaller than strongly connected components.

The paper is structured as follows: First, Section 2 introduces the main definitions and notations used throughout the paper. Section 3 presents the central notion of a modular organisation of a network along with its structural and dynamical properties. Section 4 defines elementary modular organisation and the conditions leading to obtain it. Some concluding remarks and perspectives are provided in Section 5 .

\section{The interaction network and its associated dynamics}

This section introduces the discrete based asynchronous dynamics, modelling the dynamics of biological networks.

Relation. First, we introduce basic notations. Let $\rightarrow \subseteq S \times S$ be a binary relation on a set $S$, given $s, s^{\prime} \in S$ and $S^{\prime} \subseteq S$, we denote by $s \rightarrow s^{\prime}$ the fact that $\left(s, s^{\prime}\right) \in \rightarrow$, by $(s \rightarrow) \triangleq\left\{s^{\prime} \mid s \rightarrow s^{\prime}\right\}$ the image of $s$ by $\rightarrow$, and by $\left(S^{\prime} \rightarrow\right)$ its generalisation to the state set $S^{\prime}$. Similarly, we denote by $(\rightarrow s)$ and $\left(\rightarrow S^{\prime}\right)$ the corresponding preimages. The composition of two binary relations will be denoted by $\rightarrow \circ \rightarrow^{\prime}$ and the reflexive and transitive closure by $\rightarrow^{*}=\bigcup_{i \in \mathbb{N}} \rightarrow^{i}$, with $\rightarrow^{0}$ as the identity relation.

States and operations on states. Given a set $A=\left\{a_{1}, \ldots, a_{n}\right\}$ of agents of interest, each $a_{i} \in A$ has a local state, denoted by $s_{a_{i}}$, taking values in some nonempty finite set $S_{a_{i}}$. In the examples, all $S_{a_{i}}$ are Boolean sets $\{0,1\}$, but the proposed framework is not restricted to it. A state of $A$ (or a configuration) is defined as a vector $s \in S$ associating to each $a_{i} \in A$ a value in $S_{a_{i}}$, where $S \triangleq S_{a_{1}} \times \ldots \times S_{a_{n}}$ is the set of all possible states. For any $X \subseteq A$ and $s \in S$, we denote by $\left.s\right|_{X}$ the restriction of $s$ to the agents in $X$, and by $\left.s\right|^{X}$ the completion of $s$ by all the values of agents in $X$; these notations extend to sets of states naturally. For example, the completion of the state $s_{a_{2}}=0$ by the set of agents $\left\{a_{1}, a_{3}\right\}$ is $\left.s_{a_{2}}\right|^{\left\{a_{1}, a_{3}\right\}}=\{000,001,100,101\}$, and the restriction of $s=101, s \in S_{a_{1}} \times S_{a_{2}} \times S_{a_{3}}$, on $\left\{a_{2}\right\}$ is 0 . The $X$-equivalence defines an 
equivalence relation on states with regard to the state restriction on the agent set $X$.

Definition 1. Two states $s_{1}, s_{2} \in S$ are said to be $\mathrm{X}$-equivalent and denoted by $s_{1} \sim_{X} s_{2}$, for some $X \subseteq A$, if and only if $\left.s_{1}\right|_{X}=\left.s_{2}\right|_{X}$, i.e., if they cannot be distinguished in $\left.S\right|_{X}$.

Evolution and asynchronous dynamics. An evolution is a relation on states $\rightarrow$. Each $s \rightarrow s^{\prime}$ is a transition meaning that $s$ evolves to $s^{\prime}$ by $\rightarrow$. Thus, the global evolution of $\eta$ can be represented by a directed graph $\mathcal{G}=(S, \rightarrow)$ called the state graph. In this work, we pay particular attention to local evolutions, since each agent $a \in A$ has its own evolution $\rightarrow_{a}$. The collection of all these local evolutions results in the asynchronous view of the global evolution of $\eta$, i.e., $\rightarrow=\bigcup_{a \in A} \rightarrow_{a}$.

Definition 2. The asynchronous dynamics (or dynamics for short) of a network $\eta$ is the triple $\left\langle A, S,\left(\rightarrow_{a}\right)_{a \in A}\right\rangle$, where $A$ is a set of agents, $S$ is a set of states, and for each $a \in A, \rightarrow_{a} \subseteq S \times S$ is a total or empty relation characterising the evolution of agent a such that for any $s \rightarrow_{a} s^{\prime}$, either $s=s^{\prime}$ or $s$ differs from $s^{\prime}$ only on the a-th component.

Interaction network and interaction graph. We are now in a position to introduce formally the interaction network as a family of functions $\eta=\left\{\eta_{a}\right\}_{a \in A}$, such that each $\eta_{a}: S \rightarrow S_{a}$ defines the next state $\eta_{a}(s)$ with respect to the asynchronous evolution of $a$ from $s$. Network $\eta$ allows to deduce a directed interaction graph $G \triangleq(A, \longrightarrow)$ such that $a_{i} \longrightarrow a_{j}$ if $a_{i}$ occurs in the definition of $\eta_{a_{j}}$. When $\rightarrow_{a}$ is empty for some $a$ (i.e., the local state of $a$ remains invariant), then $a$ plays the role of an input, which means that no other agents of $A$ influence it (i.e., there are no arcs towards $a$ in $G$ ), see Figure 1.

Orbit and equilibrium. Given a set $S^{\prime} \subseteq S$, we introduce the following notions:

- an orbit of $S^{\prime}, \Omega\left(S^{\prime}\right)$, is the set of states comprising $S^{\prime}$ and all the states reachable from $S^{\prime}$ by $\rightarrow$;

- an equilibrium $e \in S$ is a state reachable infinitely often by $\rightarrow$; $\Psi\left(S^{\prime}\right)$ denotes the set of equilibria reachable from $S^{\prime}$;

- an attractor is a set of equilibria $E \subseteq S$ such that $\forall e \in E: \Psi(\{e\})=E$. In a state graph, an attractor is the set of states comprised in one terminal strongly connected component ${ }^{3}$ that can be of two kinds:

\footnotetext{
${ }^{3}$ Recall that, in a terminal strongly connected component each path starting from a vertex of the component remains in this component.
} 

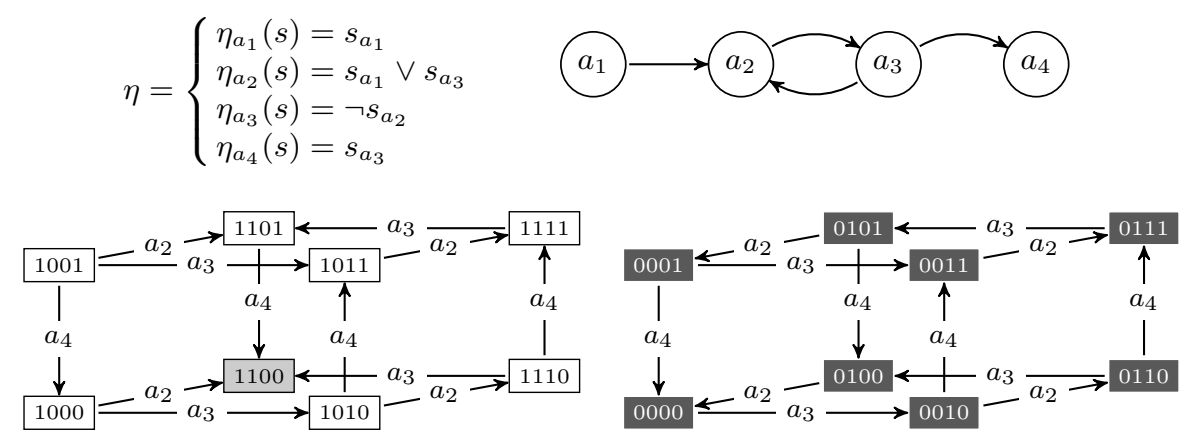

Fig. 1. An interaction network $\eta$ (top left), its graphical representation (top right), and the state graph $\mathcal{G}$ of $\eta$ composed of two disconnected components (bottom). $\mathcal{G}$ represents the dynamics of $\eta$ for each state $s \in\{0,1\}^{4}$, in which by convention, self loops are omitted, stable states are depicted in gray while limit sets are in black.

- a stable state is a singleton $E \subseteq S$;

- a limit set is an attractor $E$ such that $|E|>1$.

Moreover, the restriction of $\rightarrow$ to $X \subseteq A$ is defined as: $\rightarrow_{X}=\bigcup_{a \in X} \rightarrow_{a}$. Orbits and equilibria are determined by two operators having two arguments, an agent set and a state set.

Definition 3. The orbit operator $\Omega$ and the equilibrium operator $\Psi$, are defined as follows for $X \subseteq A$ and $S^{\prime} \subseteq S$ :

$-\Omega_{X}\left(S^{\prime}\right)=\left(S^{\prime} \rightarrow_{X}^{*}\right)$

$-\Psi_{X}\left(S^{\prime}\right)=\left\{s \in \Omega_{X}\left(S^{\prime}\right) \mid \forall s^{\prime} \in S: s \rightarrow_{X}^{*} s^{\prime} \Longrightarrow s^{\prime} \longrightarrow_{X}^{*} s\right\}$.

The equilibrium operator $\Psi_{X}$ is idempotent, upper-continuous and monotone (see Proposition 2 in Appendix).

The example in Figure 1 illustrates the dynamics of an interaction network. It is defined by $\eta$, each $\eta_{a}$ being the local transition function of agent $a$. Given a state $s$, the evolution $s \rightarrow_{a} s^{\prime}$ means that $s^{\prime}$ is obtained by applying $\eta_{a}$ to $s$, i.e., $s \rightarrow_{a} s^{\prime} \triangleq s_{a}^{\prime}=\eta_{a}(s) \wedge\left(\forall a^{\prime} \in A \backslash\{a\}: s_{a^{\prime}}^{\prime}=s_{a^{\prime}}\right)$. Let us remark that:

- the orbit of $\{1111\}$ is $\Omega(\{1111\})=\{1111,1101,1100\}$;

- 1100 is an equilibrium, as well as 0101 and 0010 are;

- the set of equilibria reachable from 0000 is $\{0 x y z \mid x, y, z \in\{0,1\}\}$;

- two attractors exist: a stable state $\{1100\}$ and a limit set $\Psi(\{0000\})$.

Regulation. The regulation is a sub-relation of the interaction specifying a dynamics-based dependence between two agents. Agent $a_{k}$ regulates 


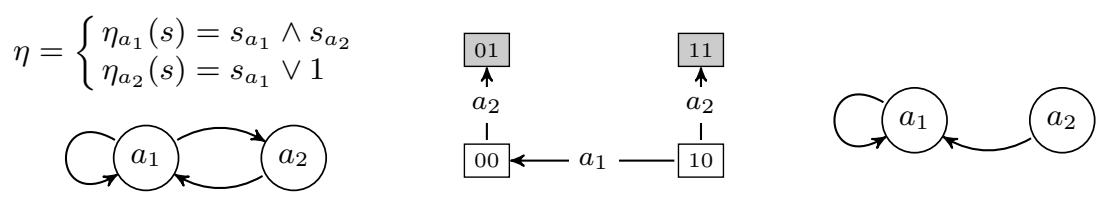

Fig. 2. An interaction network, its state graph and the corresponding regulation graph.

agent $a_{\ell}$, if at least one modification of a state of $a_{\ell}$ requires a modification of a state of $a_{k}$.

Definition 4. an interaction, $a_{k} \longrightarrow a_{\ell}$ is a regulation if and only if there exist two states $s, s^{\prime} \in S$ such that $\left(s \sim_{A \backslash\left\{a_{k}\right\}} s^{\prime}\right) \wedge\left(\left(s \rightarrow_{a_{\ell}}\right) \nsim_{a_{\ell}}\right.$ $\left.\left(s^{\prime} \rightarrow_{a_{\ell}}\right)\right)$. By extension, given $X_{i}, X_{j} \subseteq A, X_{i} \longrightarrow X_{j}$ if and only if $\exists a_{k} \in X_{i}, \exists a_{\ell} \in X_{j}: a_{k} \longrightarrow a_{\ell}$.

It may arise that the graph of interaction differs from the graph of regulation because the interaction depends on the syntactic definition of a network whereas the regulation relies on a property of the dynamics. In Figure 1, the sets of regulators of agents $a_{1}, a_{2}, a_{3}$ and $a_{4}$ are respectively $\left\{a_{1}\right\},\left\{a_{1}, a_{3}\right\},\left\{a_{2}\right\}$ and $\left\{a_{3}\right\}$. Notice also that there are the following relations on sets of agents: $\left\{a_{1}, a_{2}\right\} \longrightarrow\left\{a_{3}, a_{4}\right\},\left\{a_{3}\right\} \longrightarrow\left\{a_{2}, a_{4}\right\}$ and $\left\{a_{1}\right\} \longrightarrow\left\{a_{2}, a_{3}\right\} \longrightarrow\left\{a_{4}\right\}$. Another example of a regulation graph is given in Figure 2 (right). It shows that interaction $\left(a_{1}, a_{2}\right)$ in the interaction network is actually not a regulation because no modification of $a_{1}$ influences the state of $a_{2}$. All other interactions are effective, meaning that the underlying regulation graph contains all interactions from the network but $\left(a_{1}, a_{2}\right)$.

\section{Composition of equilibria}

In this section, a relation between the equilibria of an interaction network and that of its parts is presented. It allows to consider a modular view of the system in which each part is seen as a module, i.e., a subset of agents. It means that modules, which influence each other, reveal the underlying biological functions materialised by their equilibria.

\subsection{Modular organisation}

Our objective is to find a decomposition of the set of agents $A$ into modules, i.e., a partition ${ }^{4}$ of $A$, together with a composition operator $\oslash$ of the

\footnotetext{
${ }^{4}$ A partition of a set $A$ is a set of nonempty disjoint subsets of $A$ which covers $A$.
} 

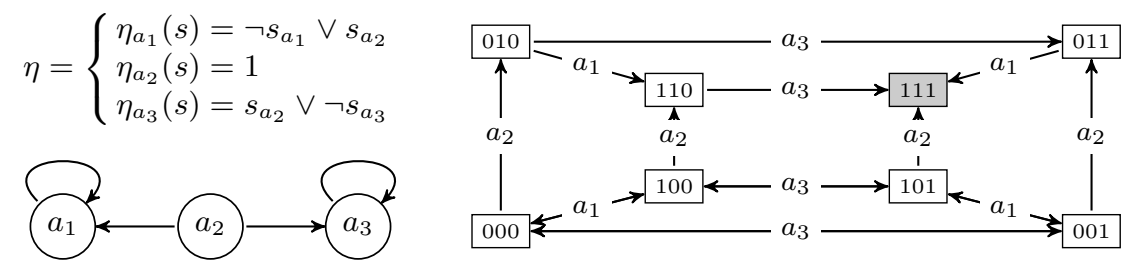

Fig. 3. An interaction network and its state graph.

equilibria of these modules, allowing to retrieve the global equilibria of the network. Finding an adequate operator $\oslash$ is a challenging question. Basically, a modular organisation $\left(X_{1}, \ldots, X_{m}\right)$ should satisfy the following equation characterizing the composition of the module equilibria:

$$
\Psi_{X_{1}} \oslash \ldots \oslash \Psi_{X_{m}}=\Psi_{\bigcup_{i=1}^{m} X_{i}}
$$

One can easily see that, in general, taking $\oslash=\cup$ for example is not a solution. If we consider the interaction network in Figure 3 and a partition into two parts $\left\{a_{1}, a_{2}\right\}$ and $\left\{a_{3}\right\}$, then the corresponding sets of equilibria are respectively $\{110,111\}$ and $\{000,001,100,101,011,111\}$, while the set of global equilibria is $\{111\}$, which is not the union of the previous ones. However, one may see that, for the same parts, the computation of the equilibria of $\left\{a_{3}\right\}$ from the equilibria of $\left\{a_{1}, a_{2}\right\}$, gives the expected property $\Psi_{\left\{a_{3}\right\}} \circ \Psi_{\left\{a_{1}, a_{2}\right\}}=\Psi_{\left\{a_{1}, a_{2}, a_{3}\right\}}$, whereas $\Psi_{\left\{a_{1}, a_{2}\right\}} \circ \Psi_{\left\{a_{3}\right\}} \neq \Psi_{\left\{a_{1}, a_{2}, a_{3}\right\}}$. This suggests that the order in which parts are taken into account plays an important role in the definition of the composition operator. Unfortunately, in general none of the usual operators such as $\cup, \cap$ and $\circ$ can be used as the modular composition operator as one can check in the following network: $\left\{\eta_{a_{1}}(s)=s_{a_{1}} \wedge \neg s_{a_{3}}, \eta_{a_{2}}(s)=s_{a_{2}} \wedge \neg s_{a_{3}}, \eta_{a_{3}}(s)=s_{a_{2}}\right\}$, while a modular decomposition exists: $\left\{a_{1}\right\}$ followed by $\left\{a_{2}, a_{3}\right\}$.

Thus, we will focus on an ordered partition $\pi=\left(X_{1}, \ldots, X_{m}\right)$ of $A$, i.e., a partition of $A$ provided with a strict total order and represented by a sequence, called a modular organisation, preserving (1). Furthermore, we would like to be able to "fold" contiguous modules in $\pi$ in order to deal with them as with a single module ${ }^{5}$, while preserving the result of the composition of equilibria. As a consequence, we require a modular organisation to support folding and to be such that the composition operator $\oslash$ is associative according to the order in $\pi$.

In order to form a modular organisation, the modules and their order in $\pi$ should satisfy some conditions related to their dynamics. Intuitively, two

\footnotetext{
${ }^{5}$ The folding of modules corresponds to the union of these modules.
} 
disjoint sets of agents $X_{i}$ and $X_{j}, i<j$, can be modules in $\pi$, either if they do not regulate each other, or if $X_{i}$ regulates $X_{j}$. In both cases, we can remark that the equilibria of $X_{i}$ should embed the asymptotic evolution of $X_{j}$, which leads to encompass the equilibria of $X_{j}$ in the equilibria of $X_{i}$. These conditions are expressed by the modularity relation ( $M$-relation).

Definition 5. The $M$-relation $\rightsquigarrow \subseteq \mathcal{P}(A) \times \mathcal{P}(A)$ is defined as:

$$
X_{i} \rightsquigarrow X_{j} \triangleq \forall S^{\prime} \subseteq S:\left(\Psi_{X_{i}} \circ \Psi_{X_{i} \cup X_{j}}\left(S^{\prime}\right)\right) \rightarrow_{X_{j}} \subseteq\left(\Psi_{X_{i}} \circ \Psi_{X_{i}} \cup X_{j}\left(S^{\prime}\right)\right) .
$$

Some fundamental properties of the $M$-relation can be found in Proposition 3 of the Appendix. In this context, a modular organisation can be defined as follows.

Definition 6. A modular organisation $\left(X_{1}, \ldots, X_{m}\right)$ is an ordered partition of $A$ such that for all $1<i \leq m:\left(\bigcup_{j=1}^{i-1} X_{j}\right) \rightsquigarrow X_{i}$.

From Definition 6, Proposition 1 states that being a modular organisation is preserved by any folding of its contiguous parts ${ }^{6}$.

Proposition 1. Let $\pi=\left(X_{1}, \ldots, X_{m}\right)$ be a modular organisation. For all $1 \leq i \leq j \leq m,\left(X_{1}, \ldots, X_{i-1}, \bigcup_{k=i}^{j} X_{k}, X_{j+1}, \ldots, X_{m}\right)$ is a modular organisation.

In the literature $[6,10,11]$, modules are frequently assimilated to SCCs of interaction networks. Although these works focus on structural arguments only, it turns out that they are compatible with Definition 6. Indeed, any topological order ${ }^{7}$ of SCCs is actually a modular organisation. Notice that, a topological order on the quotient graph of SCCs always exist since the graph is acyclic. For instance, $\left(\left\{a_{1}\right\},\left\{a_{2}, a_{3}\right\},\left\{a_{4}\right\}\right)$ is a modular organisation of the interaction network presented in Figure 1. In what follows, we present an approach addressing formally this aspect. As a result, we show that, in particular, the structural decomposition in SCCs makes sense and may be improved by a deeper analysis leading to the decomposition of SCCs in elementary modules (see Section 4), potentially smaller than those coming from SCCs.

\subsection{Regulation and modularity relation}

The regulation and the $M$-relation are related, as shown below.

Lemma 1. For any $X_{i}, X_{j}$ subsets of $A: \neg\left(X_{j} \longrightarrow X_{i}\right) \Longrightarrow X_{i} \rightsquigarrow X_{j}$.

\footnotetext{
${ }^{6}$ Proofs are in Appendix.

${ }^{7}$ A topological order is a total order obtained by topological sorting.
} 


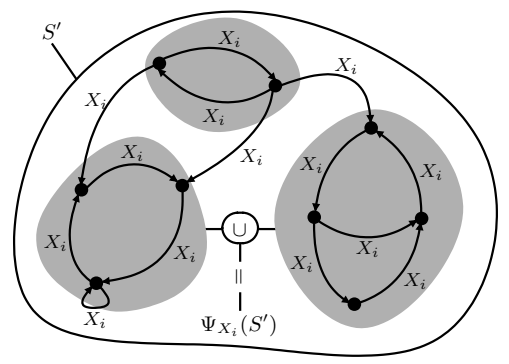

(a)

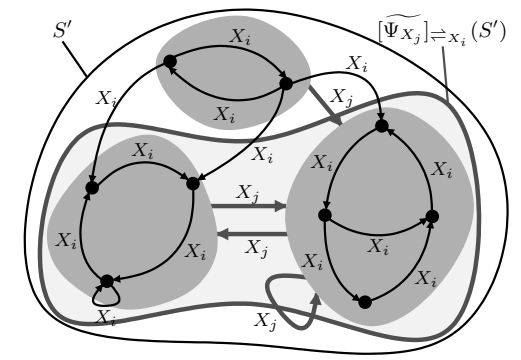

(b)

Fig. 4. Successive steps leading to the definition of the composition operator $\oslash$. The SCCs defined for $\rightarrow_{X_{i}}$ are in gray (a). The two terminal SCCs at the bottom correspond to attractors of $\rightarrow_{X_{i}}$ (a). The equilibria $\Psi_{X_{i} \cup X_{j}}\left(S^{\prime}\right)$ is computed from $\rightarrow_{X_{j}}$ (bold $X_{j}$ arrows) on these attractors (b).

According to Definition 6, Theorem 1 provides a connection between structural properties of a regulation graph and the corresponding modular organisations (possibly reduced to a single module).

Theorem 1. Any topological order of the SCC quotient graph of a regulation graph is a modular organisation.

\subsection{Composition operator}

In this section, we present the successive steps leading to the definition of the composition operator $\oslash$. From (1), $\oslash$ is a binary operator that applies on the equilibria of parts $X_{i}$ and $X_{j}$ of $\pi$, with $i<j$. Thus, its definition is based on the attractors of $\rightarrow_{X_{i}}$ which correspond to terminal nodes (terminal SCCs) of the SCC quotient graph of $\rightarrow_{X_{i}}$, namely $\mathcal{G} / \rightleftharpoons_{X_{i}}$, where $\rightleftharpoons_{X_{i}}$ is the equivalence relation identifying states belonging to the same SCC and defined as $s \rightleftharpoons X_{i} s^{\prime} \triangleq\left(s \rightarrow_{X_{i}}^{*} s^{\prime}\right) \wedge\left(s^{\prime} \rightarrow_{X_{i}}^{*} s\right)$. For any $S^{\prime} \subseteq S$, an attractor of $\rightarrow_{X_{i}}$ coincides with $[s]_{X_{i}} \subseteq \Psi_{X_{i}}\left(S^{\prime}\right)$ (see Figure 4.a). Moreover, for all $S^{\prime} \subseteq S$, we denote by:

$-\left[S^{\prime}{\rightleftharpoons_{X_{i}}}=\left\{[s]_{\chi_{X}} \mid s \in S^{\prime}\right\}\right.$ the set of equivalence classes of $\rightleftharpoons_{X_{i}}$ in $S^{\prime}$;

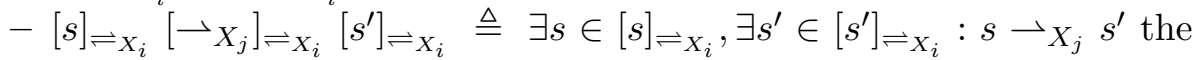
evolution by agents of $X_{j}$ on these equivalence classes.

We define an operator $\left[\Psi_{X_{j}}\right]_{\rightleftharpoons_{X_{i}}}$, similar to the equilibria operator, computing the set of equilibria of $\left[-_{X_{j}}\right]_{\rightleftharpoons_{X_{i}}}$ in $\mathcal{G} / \rightleftharpoons_{X_{i}}$ (see Figure 4 .b and Section 3.4 for the algorithm) as follows:

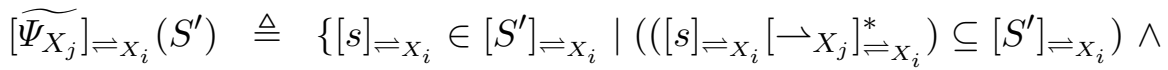

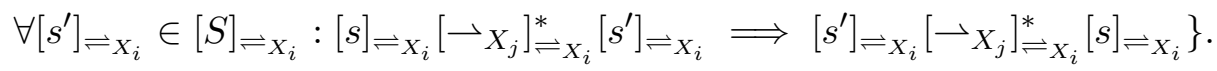


Operator $\oslash$ is thus defined as:

$$
\Psi_{X_{i}} \oslash \Psi_{X_{j}} \triangleq \text { Flat } \circ\left[\widetilde{\Psi_{X_{j}}}\right]_{\rightleftharpoons_{X_{i}}} \circ \Psi_{X_{i}},
$$

where, for any set $E \subseteq \mathcal{P}(S)$, Flat $(E)=\bigcup_{e \in E} e$ flattens the set. Thus, if applied to a set of attractors, Flat gives the underlying set of equilibria. For example, Flat $(\{\{00,01\},\{10\}\})=\{00,01,10\}$. As a result, one can see that $\oslash$ does compute the set of states belonging to the attractors of $X_{i}$ which are also the equilibria of $\rightarrow_{X_{j}}$.

Lemma 2 below shows that the global equilibria of $X_{i} \cup X_{j}$ are obtained using the composition $\Psi_{X_{i}} \oslash \Psi_{X_{j}}$.

Lemma 2. For all $X_{i}, X_{j}$ disjoint subsets of $A$ :

$$
X_{i} \rightsquigarrow X_{j} \Longrightarrow \forall S^{\prime} \subseteq S: \Psi_{X_{i} \cup X_{j}}\left(S^{\prime}\right)=\left(\Psi_{X_{i}} \oslash \Psi_{X_{j}}\right) \circ \Omega_{X_{i} \cup X_{j}}\left(S^{\prime}\right) .
$$

As a main result, the computation of global equilibria of an interaction network can be obtained modularly (Theorem 2).

Theorem 2. Let $A^{\prime}=\bigcup_{i=1}^{m} X_{i} \subseteq A$ be a set of agents, if $\left(X_{1}, \ldots, X_{m}\right)$ is a modular organisation then we have:

$$
\Psi_{A^{\prime}}=\left(\Psi_{X_{1}} \oslash \ldots \oslash \Psi_{X_{m}}\right) \circ \Omega_{A^{\prime}} .
$$

\subsection{Modular and incremental computation of equilibria}

Modularity allows an incremental and efficient computation of the equilibria avoiding the generation of the complete state space $S$. The algorithm presented in Figure 5 is an application of Theorem 2 introducing incremental processing based on the fact that the evolution of an agent only depends on the current states of its regulators. Indeed, the equilibria of a set of agents $X$ in a state space $\left.S\right|_{X \cup Y}$ where $X$ and $Y$ are disjoint sets and $X \cup Y$ contains the regulators $R_{X}=(\longrightarrow X)$ of $X$, can be computed from the restriction to $X \cup R_{X}$ completed by $Y \backslash R_{X}$. In other words, the equilibria computation operator and the completion operator commute for an appropriate selection of sets of agents, as shown by the following lemma.

Lemma 3. $\Psi_{X}\left(\left.S\right|_{X \cup Y}\right)=\left.\Psi_{X}\left(\left.S\right|_{X \cup R_{X}}\right)\right|^{Y \backslash R_{X}}$, with $R_{X}=(\longrightarrow X), R_{X} \subseteq X \cup Y$ and $X \cap Y=\emptyset$.

Each step of the algorithm is seen as the computation of equilibria for the following modular organisation $\left(X, X_{i}\right)$ where $X=X_{1} \cup \ldots \cup X_{i-1}$ is the 
folding of the modules preceding $X_{i}$ in the initial modular organisation and corresponds to the following equation:

$$
\Psi_{X \cup X_{i}}(S)=\text { Flat }\left(\left[\widetilde{\Psi_{X_{i}}}\right]_{\rightleftharpoons_{X}}\left(\left.\Psi_{X}\left(\left.S\right|_{X \cup R_{X}}\right)\right|^{X_{i} \backslash R_{X}}\right)\right) .
$$

The algorithm of Figure 5 is divided in two parts: PART I corresponding to the completion of states by $X_{i}$, and PART II corresponding to the computation of the attractors taking into account $\rightarrow_{X_{i}}$. In PART I the attractors are duplicated by completing their state values while preserving the structure of attractors. PART II is divided into two subparts. PART II.1 computes the quotient graph with attractors as vertices and the quotiented evolution by $X_{i}$ as arcs. Notice that some attractors are removed during this step. Indeed, from some states belonging to attractors, $\rightarrow_{X_{i}}$ may reach states located outside the attractors set. By definition of the $M$-relation (Definition 5), they cannot be considered as equilibria and are not included. The remaining set of attractors, called the core, is used in PART II.2 to compute the equilibria as the terminal SCCs of the core graph. Since TermSCCs returns a set of "attractors of attractors", they are finally flattened to retrieve the structure of a set of equilibria.

The complexity of the algorithm in the product of the maximal number of equilibria by the number of agents is exponential in general. It is however linear for acyclic regulatory graphs. Let $\alpha_{i}$ be the number of equilibria computed at step $i$, and $N$ the set of newly introduced agents. The computation time is bounded by $k \cdot \sum_{i=1}^{m} \alpha_{i} \cdot 2^{|N|}$, for $k \in \mathbb{N}$. The exponential time corresponds to the computation of the completion and of the quotient graph. $|N|$ is bounded by $\beta$, the number of agents in the greatest SCC, under the assumption that all modular organisations are subdivisions of topological orders of the SCC quotient graphs. Indeed, in the modular organisation the regulators of $X_{i}$ always precede $X_{i}$ unless they are also regulated by $X_{i}$. Hence, the computation time is bounded by $k \cdot 2^{\beta} \cdot m \cdot \alpha$ where $\alpha$ stands for the maximal number of equilibria for all steps, leading to a complexity in $\mathcal{O}\left(2^{\beta} \cdot m \cdot \alpha\right)$.

In the worst case, notably corresponding to a regulatory graph reduced to a single SCC, the complexity is the same as the brute-force algorithm computing equilibria from the whole state graph (i.e., $\pi=\{A\}, \beta=|A|$ ). The algorithm is more efficient in practice. In particular, for networks whose interaction graph is acyclic, each module corresponds to a single agent $(\beta=1)$ leading to a complexity in $\mathcal{O}(|A| \cdot \alpha)$ with $\alpha \leq 2^{\Delta_{0}}$ where $\Delta_{0}$ is the number of all input agents having no regulators but possibly themselves. Hence, for regulatory path-graphs, the algorithm is linear in the number of agents because $\Delta_{0}=1$. 
Input: $\left(X_{1}, \ldots, X_{m}\right)$ a modular organisation of $A$.

Result: the set of equilibria, $\Psi_{A}(S)$.

Function: TermSCCs $(\mathcal{G})$ computes the set of terminal strongly connected

Variables: components of a graph $\mathcal{G}$.

- $A^{\prime}$ : set of agents already processed;

$-R_{X_{i}}$ : regulators of $X_{i}$;

- $N$ : set of new agents;

$-\tilde{\psi}, \tilde{\psi}_{t m p}:$ set of attractors;

- $\mathcal{G}$ : quotient graph with attractors as vertices.

States are encoded by words, with $\epsilon$ as the empty word.

$/ /$ initialisation

$A^{\prime}=\emptyset$;

$\tilde{\psi}=\{\{\epsilon\}\}$;

for $i=1$ to $m$ do

PART I

// Extension of attractor states

$R_{X_{i}}=\left(\longrightarrow X_{i}\right) ; / /$ regulators of $X_{i}$

$N=\left(X_{i} \cup R_{X_{i}}\right) \backslash A^{\prime} ; / /$ new agents for evolution computation

$\tilde{\psi}_{t m p}=\emptyset$;

foreach $A t t \in \tilde{\psi}$ do

// structure preserving completion of attractors ;

foreach $\left.s_{N} \in S\right|_{N}$ do $\tilde{\psi}_{t m p}=\tilde{\psi}_{t m p} \cup\left\{\left\{\left.s|s|_{A^{\prime}} \in A t t \wedge s\right|_{N}=s_{N}\right\}\right\} ;$

end

$\tilde{\psi}=\tilde{\psi}_{t m p}$

// Attractors computation

PART II

PART II.1

// Quotient graph computation with attractors as vertices

$\tilde{\psi}_{t m p}=\emptyset$;

foreach $A t t \in \tilde{\psi}$ do

// computation of the core of attractors ;

if $\left(A t t\left[\rightarrow_{X_{i}}\right]_{\rightleftharpoons_{A^{\prime}}}^{*}\right) \subseteq \tilde{\psi}$ then $\tilde{\psi}_{t m p}=\tilde{\psi}_{t m p} \cup\{A t t\} ;$

end

$\mathcal{G}=\left(\tilde{\psi}_{\text {tmp }},\left[\overrightarrow{ }_{X_{i}}\right]_{\rightleftharpoons_{A^{\prime}}}\right) ; / /$ quotient graph of $\rightarrow_{X_{i}}$ defined on the core

$\tilde{\psi}_{\mathcal{G}}=\operatorname{TermSCCs}(\mathcal{G}) ; / /$ equilibria computation on $\mathcal{G}$

PART II. 2

// Computation of the set of attractors for $X_{1}, \ldots, X_{i}$

$\tilde{\psi}=\emptyset$

foreach $A t t \in \tilde{\psi}_{\mathcal{G}}$ do $\tilde{\psi}=\tilde{\psi} \cup\{\operatorname{Flat}($ Att $)\} ; / /$ flatten each attractor $\rightarrow \tilde{\psi}$ $A^{\prime}=A^{\prime} \cup X_{i}$;

end

return Flat $(\tilde{\psi}) ; / /$ flatten the attractor set $\rightarrow$ equilibria set

Fig. 5. Algorithm of modular and incremental computation of equilibria 
A modular organisation based on SCCs is computed by first identifying the quotient graph of SCCs and then obtaining a topological order, whose complexity is in $\mathcal{O}\left(|A|^{2}\right)$.

\section{Elementary modular organisation}

Informally, a module is elementary if it is not separable, i.e., if the equilibria of each of its agents depend entirely on the equilibria of all the others. For instance, consider negative circuits that lead to asymptotic sustained oscillations [21]. In such regulation patterns, the equilibria of an agent cannot be encompassed into that of the others because, in order to reach its own equilibria, each agent evolves from the equilibria of all the others.

In this context, a modular organisation provided by some topological orders of the SCC quotient graph (see Theorem 1) does not always provide an elementary decomposition.

Figure 6 depicts an interaction network $\eta$ composed of three agents $a_{1}$, $a_{2}$ and $a_{3}$ with the associated strongly connected regulation graph. Its underlying state graph shows that the global dynamics of $\eta$ leads to two attractors, stable state $\{111\}$ and limit set $\{000,100,101,001\}$. It is easy to see that $\left\{a_{2}\right\} \rightsquigarrow\left\{a_{1}, a_{3}\right\}$ and that this $M$-relation is (obviously) preserved by folding, because there are only two modules. Hence, ordered partition $\left(\left\{a_{2}\right\},\left\{a_{1}, a_{3}\right\}\right)$ is a modular organisation of $\eta$. However, separa-

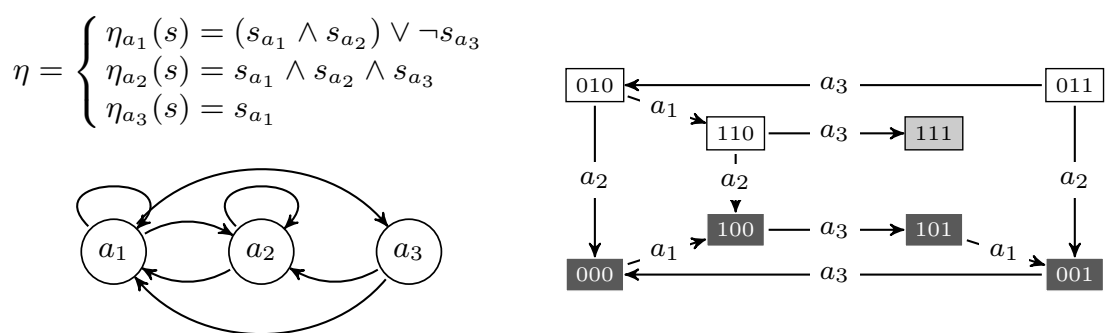

Fig. 6. A separable network with modular organisation $\left(\left\{a_{2}\right\},\left\{a_{1}, a_{3}\right\}\right)$.

bility is not possible in general as illustrated in Figure 7. Indeed, starting from modular organisation $\pi=\left(\left\{a_{1}\right\},\left\{a_{2}, a_{3}\right\}\right)$ obtained from the SCCs, the separation of $\left\{a_{2}, a_{3}\right\}$ should lead to one of the ordered partitions $\pi^{\prime}=\left(\left\{a_{1}\right\},\left\{a_{2}\right\},\left\{a_{3}\right\}\right)$ and $\pi^{\prime \prime}=\left(\left\{a_{1}\right\},\left\{a_{3}\right\},\left\{a_{2}\right\}\right)$. The condition for $\pi^{\prime}$ to be a modular organisation is that $\left\{a_{1}, a_{2}\right\} \rightsquigarrow\left\{a_{3}\right\}$, i.e., the evolution 
by $a_{3}$ from the equilibria of $\left\{a_{1}, a_{2}\right\}$ has to be included in the equilibria of $\left\{a_{1}, a_{2}\right\}$. We can observe that the attractors for $\left\{a_{1}, a_{2}\right\}$ are $\{001,101\}$ and $\{010,110\}$, while the evolution by $a_{3}$ from either 101 or 110 leaves the attractors of $\left\{a_{1}, a_{2}\right\}$, which means that $\pi^{\prime}$ is not a modular organisation. As a consequence, $\left\{a_{2}, a_{3}\right\}$ cannot be separated. Indeed, here, although agents $a_{2}$ and $a_{3}$ are together $M$-related and thus can be separated $a$ priori, they cannot be in the context of agent $a_{1}$. The same reasoning applies for $\pi^{\prime \prime}$.

Hence, the separation condition of a module $X_{i}$ in $\pi$ is not local to this module but depends on the module "context", that is the global equilibria (i.e., $\Psi_{\bigcup_{k=1}^{i-1} X_{k}}$ ) of the modules that precede. Deciding the separability of $X_{i}$ into $X_{i}^{1}$ and $X_{i}^{2}$ implies checking two conditions: $\bigcup_{k=1}^{i-1} X_{k} \rightsquigarrow X_{i}^{1}$ and $\bigcup_{k=1}^{i-1} X_{k} \cup X_{i}^{1} \rightsquigarrow X_{i}^{2}$. Of course, the complexity of the underlying computation is exponential in the size of $\pi$ and also depends on the position of $X_{i}$ in $\pi$. Nevertheless, brute-force computation may be used in practice for small interaction networks (of about 15 agents). A more efficient method allowing to go beyond this limitation is, for the moment, an open question.

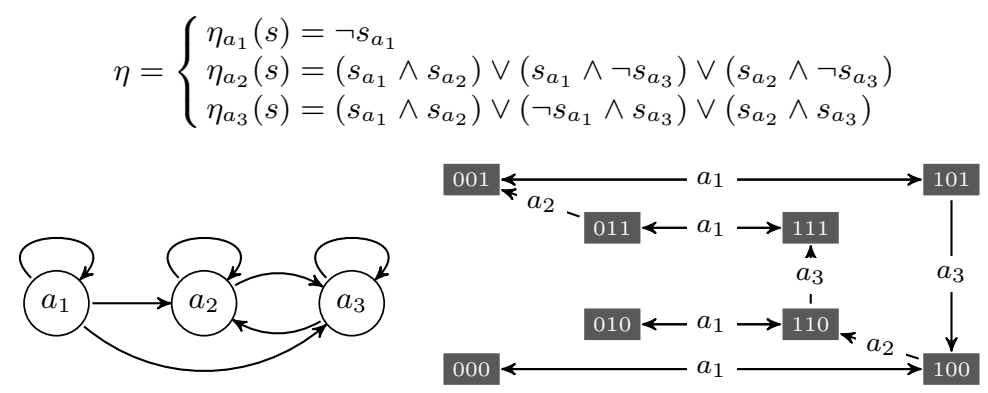

Fig. 7. Example of non-separability of $\left\{a_{2}, a_{3}\right\}$ in $\pi=\left(\left\{a_{1}\right\},\left\{a_{2}, a_{3}\right\}\right)$.

\section{Conclusion}

We developed a formal framework for the analysis of the modularity in interaction networks assuming asymptotic dynamics of modules and enabling their composition. We exhibited modularity conditions governing the composition of modules and an efficient computation method such that the global equilibria of interaction networks are obtained from the local ones, leading to an efficient algorithm. Moreover, we confirmed that 
usual assumptions identifying modules with SCCs have a strong motivation coming from theory.

The next step should be identifying a characteristic property for finding elementary modular organisations. Then, since this work provides a rigorous setting for studying other questions around modularity. For example, a success factor of synthetic biology is to ensure the safety of modular design [22] which in our theoretical framework is guaranteed by construction through the concept of modular organisation. Also, questions related to robustness and evolution could be tackled thanks to the modular knowledge of interaction networks.

\section{References}

1. Monod, J.: Chance and necessity: an essay on the natural philosophy of modern biology. Knopf (1970)

2. Delbrück, M., ed.: Viruses, California Institute of Technology (1950)

3. Lederberg, E.M., Lederberg, J.: Genetic studies of lysogenicity in Escherichia coli. Genetics 38 (1953) 51-64

4. Jacob, F., Monod, J.: Genetic regulatory mechanisms in the synthesis of proteins. Journal of Molecular Biology 3 (1961) 318-356

5. Qi, Y., Ge, H.: Modularity and dynamics of cellular networks. PLoS Computational Biology 2 (2006) e174

6. Gagneur, J., Krause, R., Bouwmeester, T., et al.: Modular decomposition of protein-protein interaction networks. Genome Biology 5 (2004) R57

7. Chaouiya, C., Klaudel, H., Pommereau, F.: A modular, qualitative modeling of regulatory networks using Petri nets. In: Modeling in systems biology: the Petri nets approach. Springer (2011) 253-279

8. Rives, A.W., Galitski, T.: Modular organization of cellular networks. Proceedings of the National Academy of Sciences of the USA 100 (2003) 1128-1133

9. Spirin, V., Mirny, L.A.: Protein complexes and functional modules in molecular networks. Proceedings of the National Academy of Sciences of the USA 100 (2003) $12123-12128$

10. Milo, R., Shen-Orr, S., Itzkovitz, S., et al.: Network motifs: simple building blocks of complex networks. Science 298 (2002) 824-827

11. Alon, U.: Biological networks: The tinkerer as an engineer. Science 301 (2003) 1866-1867

12. Bar-Joseph, Z., Gerber, G.K., Lee, T.I., et al.: Computational discovery of gene modules and regulatory networks. Nature biotechnology 21 (2003) 1337-1342

13. Segal, E., Shapira, M., Regev, A., et al.: Module networks: discovering regulatory modules and their condition specific regulators from gene expression data. Nature genetics 34 (2003) 166-176

14. Thieffry, D., Romero, D.: The modularity of biological regulatory networks. Biosystems 50 (1999) 49-59

15. Han, J.D.J.: Understanding biological functions through molecular networks. Cell Research 18 (2008) 224-237

16. Siebert, H.: Dynamical and structural modularity of discrete regulatory networks. In: Proceedings of CompMod. Volume 6 of Electronic Proceedings in Theoretical Computer Science., Open Publishing Association (2009) 109-124 
17. Delaplace, F., Klaudel, H., Cartier-Michaud, A.: Discrete causal model view of biological networks. In: Proceedings of CMSB, ACM (2010) 4-13

18. Demongeot, J., Goles, E., Morvan, M., et al.: Attraction basins as gauges of robustness against boundary conditions in biological complex systems. PLoS One 5 (2010) e11793

19. Bernot, G., Tahi, F.: Behaviour preservation of a biological regulatory network when embedded into a larger network. Fundamenta Informaticae 91 (2009) 463485

20. Thomas, R.: Boolean formalisation of genetic control circuits. Journal of Theoretical Biology 42 (1973) 563-585

21. Thomas, R.: On the relation between the logical structure of systems and their ability to generate multiple steady states or sustained oscillations. In: Numerical methods in the study of critical phenomena. Volume 9 of Springer Series in Synergetics. Springer (1981) 180-193

22. Purnick, P.E.M., Weiss, R.: The second wave of synthetic biology: from modules to systems. Nature Reviews Molecular Cell Biology 10 (2009) 410-422

\section{Appendix}

Proposition 2 (Properties of $\Psi$ ). Let $\left\langle A, S,\left(\rightarrow_{a}\right)_{a \in A}\right\rangle$ be an asynchronous dynamics and $X \subseteq A$ be a subset of agents. $\Psi_{X}$ has the following properties, for all sets $S^{\prime}, S^{\prime \prime}$ subsets of states:

a. Idempotency: $\Psi_{X} \circ \Psi_{X}\left(S^{\prime}\right)=\Psi_{X}\left(\Psi_{X}\left(S^{\prime}\right)\right)=\Psi_{X}\left(S^{\prime}\right)$;

b. Upper-continuity: $\Psi_{X}\left(S^{\prime} \cup S^{\prime \prime}\right)=\Psi_{X}\left(S^{\prime}\right) \cup \Psi_{X}\left(S^{\prime \prime}\right)$;

c. Monotony (order-preserving) : $S^{\prime} \subseteq S^{\prime \prime} \Longrightarrow \Psi_{X}\left(S^{\prime}\right) \subseteq \Psi_{X}\left(S^{\prime \prime}\right)$.

Proof (Proposition 2). Let $E q_{X}(s)$ be the predicate meaning that $s$ is an equilibrium for $\rightarrow_{X}$.

a. By expanding $\Psi_{X}\left(\Psi_{X}\left(S^{\prime}\right)\right)$, we have:

$$
\begin{aligned}
\Psi_{X}\left(\Psi_{X}\left(S^{\prime}\right)\right) & =\left\{s \in \Omega_{X}\left(\Psi_{X}\left(S^{\prime}\right)\right) \mid E q_{X}(s)\right\} \\
& =\left\{s \in \Psi_{X}\left(S^{\prime}\right) \mid E q_{X}(s)\right\} \\
& =\Psi_{X}\left(S^{\prime}\right) .
\end{aligned}
$$

b. By definition, $\Psi_{X}\left(S^{\prime} \cup S^{\prime \prime}\right)=\left\{s \in \Omega_{X}\left(S^{\prime} \cup S^{\prime \prime}\right) \mid E q_{X}(s)\right\}$, where $\Omega_{X}\left(S^{\prime} \cup S^{\prime \prime}\right)=\left(S^{\prime} \cup S^{\prime \prime}\right) \longrightarrow_{X}^{*}$. Since $\rightarrow_{X}^{*}$ is upper-continuous on the lattice of state sets, we have:

$$
\begin{aligned}
\Psi_{X}\left(S^{\prime} \cup S^{\prime \prime}\right) & =\left\{s \in \Omega_{X}\left(S^{\prime}\right) \cup \Omega_{X}\left(S^{\prime \prime}\right) \mid E q_{X}(s)\right\} \\
& =\left\{s \in \Omega_{X}\left(S^{\prime}\right) \mid E q_{X}(s)\right\} \cup\left\{s \in \Omega_{X}\left(S^{\prime \prime}\right) \mid E q_{X}(s)\right\} \\
& =\Psi_{X}\left(S^{\prime}\right) \cup \Psi_{X}\left(S^{\prime \prime}\right) .
\end{aligned}
$$

c. An upper-continuous function is monotone. 
Proposition 3 (Properties of the modularity relation). Let $S^{\prime}$ be a subset of $S$. For all $X_{i}, X_{j}$ subsets of $A$, we have the following properties:

1. $X_{i} \rightsquigarrow X_{j} \Longleftrightarrow \Psi_{X_{i} \cup X_{j}}\left(S^{\prime}\right) \subseteq\left(\Psi_{X_{i}} \circ \Omega_{X_{i} \cup X_{j}}\left(S^{\prime}\right)\right)$;

2. $X_{i} \rightsquigarrow X_{j} \Longleftrightarrow \Psi_{X_{i} \cup X_{j}}\left(S^{\prime}\right)=\Psi_{X_{i}} \circ \Psi_{X_{i} \cup X_{j}}\left(S^{\prime}\right)$.

Proof. 1. $(\Rightarrow)$ Let $S^{\prime} \subseteq S, s \in \Psi_{X_{i} \cup X_{j}}\left(S^{\prime}\right)$ and $s \notin \Psi_{X_{i}}\left(\Omega_{X_{i} \cup X_{j}}\left(S^{\prime}\right)\right)$, and $s^{\prime} \in \Psi_{X_{i}}\left(\Omega_{X_{i} \cup X_{j}}(\{s\})\right)$. By definition of equilibrium, $s^{\prime} \rightarrow_{X_{i} \cup X_{j}}^{*} s$. Now, we have:

$-\forall s^{\prime \prime} \in\left(s^{\prime} \rightarrow_{X_{i}}\right): s^{\prime \prime} \in \Psi_{X_{i}} \circ \Psi_{X_{i} \cup X_{j}}\left(\left\{s^{\prime}\right\}\right)$, by definition of equilibria;

$-\forall s^{\prime \prime} \in\left(s^{\prime} \rightarrow_{X_{j}}\right): s^{\prime \prime} \in \Psi_{X_{i}} \circ \Psi_{X_{i} \cup X_{j}}\left(\left\{s^{\prime}\right\}\right)$, by definition of $\rightsquigarrow$.

As a consequence, $s^{\prime} \mathcal{-}_{X_{i}}^{*} \cup X_{j} s$, which leads to a contradiction.

$(\Leftarrow)$ Let $S^{\prime} \subseteq S, s \in \Psi_{X_{i} \cup X_{j}}\left(S^{\prime}\right)$, and $\Psi_{X_{i} \cup X_{j}}\left(S^{\prime}\right) \subseteq \Psi_{X_{i}}\left(\Omega_{X_{i} \cup X_{j}}\left(S^{\prime}\right)\right)$.

Then, by hypothesis, we have:

$$
\forall s^{\prime \prime} \in\left(s \rightarrow_{X_{j}}\right): s^{\prime \prime} \in \Psi_{X_{i} \cup X_{j}}\left(S^{\prime}\right) \wedge s^{\prime \prime} \in \Psi_{X_{i}}\left(\Omega_{X_{i} \cup X_{j}}\left(S^{\prime}\right)\right),
$$

which means that $s, s^{\prime \prime} \in \Psi_{X_{i}} \circ \Psi_{X_{i} \cup X_{j}}\left(S^{\prime}\right)$. Thus, $X_{i} \rightsquigarrow X_{j}$.

2. From Proposition 3.1 and since $\Psi_{X_{i} \cup X_{j}}\left(S^{\prime}\right)=\Omega_{X_{i} \cup X_{j}}\left(\Psi_{X_{i} \cup X_{j}}\left(S^{\prime}\right)\right)$.

Proof (Proposition 1). Let $\pi=\left(X_{1}, \ldots, X_{i-1}, X_{i}, X_{i+1}, \ldots, X_{m}\right)$ be a modular organisation and let $X=\bigcup_{k=1}^{i-1} X_{k}$. We want to show that $\left(X_{1}, \ldots, X_{i-1}, X_{i} \cup X_{i+1}, \ldots, X_{m}\right)$ is a modular organisation. By definition 6 , we have:

$$
\left(X \cup X_{i}\right) \rightsquigarrow X_{i+1}
$$

and:

$$
X \rightsquigarrow X_{i} .
$$

We want to show that: $(4) \wedge(5) \Longrightarrow X \rightsquigarrow\left(X_{i} \cup X_{i+1}\right)$. First, by Proposition 3.2, we can write:

$$
\begin{gathered}
\Psi_{X \cup X_{i}} \circ \Psi_{X \cup X_{i} \cup X_{i+1}}=\Psi_{X \cup X_{i} \cup X_{i+1}} \text { by (4), } \\
\Psi_{X} \circ \Psi_{X \cup X_{i}}=\Psi_{X \cup X_{i}} \text { by }(5) .
\end{gathered}
$$

Thus:

$$
\begin{aligned}
\Psi_{X} \circ \Psi_{X \cup X_{i} \cup X_{i+1}} & =\Psi_{X} \circ\left(\Psi_{X \cup X_{i}} \circ \Psi_{X \cup X_{i} \cup X_{i+1}}\right) \text { by }(6) \\
& =\left(\Psi_{X} \circ \Psi_{X \cup X_{i}}\right) \circ \Psi_{X \cup X_{i} \cup X_{i+1}} \\
& =\Psi_{X \cup X_{i}} \circ \Psi_{X \cup X_{i} \cup X_{i+1}} \text { by }(7) \\
& =\Psi_{X \cup X_{i} \cup X_{i+1}} \text { by }(6),
\end{aligned}
$$

which is the expected result. From Proposition 3.2, we can deduce that: $X \rightsquigarrow\left(X_{i} \cup X_{i+1}\right)$. Iteratively, we show that $X \rightsquigarrow \bigcup_{k=i}^{j} X_{k}$. As a result, $\left(X_{1}, \ldots, X_{i-1}, \bigcup_{k=i}^{j} X_{k}, X_{j+1}, \ldots, X_{m}\right)$ is a modular organisation. 
Proof (Lemma 1). By Definition 4 , for any $X_{i}, X_{j} \subseteq A$ and for any $s, s^{\prime} \in$ $S$, we have $\neg\left(X_{j} \longrightarrow X_{i}\right) \wedge\left(s \sim_{A \backslash X_{j}} s^{\prime}\right) \Longrightarrow\left(s \rightarrow_{X_{i}}\right) \sim_{X_{i}}\left(s^{\prime} \longrightarrow_{X_{i}}\right)$. This property is obviously preserved at equilibria. Indeed, for any $s, s^{\prime} \in$ $S$, we have $\neg\left(X_{j} \longrightarrow X_{i}\right) \wedge\left(s \sim_{A \backslash X_{j}} s^{\prime}\right) \Longrightarrow \Psi_{X_{i}}(s) \sim_{X_{i}} \Psi_{X_{i}}\left(s^{\prime}\right)$. Thus, the restrictions $\Psi_{X_{i}}(s)$ and $\Psi_{X_{i}}\left(s^{\prime}\right)$ to $X_{i}$ are identical. Then, the evolution by $X_{j}$ from the equilibria of $X_{i}$ remains in the equilibria of $X_{i}$. Hence, we get that $\neg\left(X_{j} \longrightarrow X_{i}\right) \Longrightarrow X_{i} \rightsquigarrow X_{j}$.

Proof (Theorem 1). Observe that, in the SCC quotient graph $G$ of a regulation graph, $X_{i} \longrightarrow X_{j}$ always implies that $\neg\left(X_{j} \longrightarrow X_{i}\right)$, because of the acyclicity of $G$. Thus, folding contiguous modules with respect to any topological order preserves the absence of regulation. As a consequence, if $\left(X_{1}, \ldots, X_{m}\right)$ is a topological order of $G$, for all $i, j \in \mathbb{N}$ such that $1 \leq i \leq j \leq m$, we have $\neg\left(X_{j} \longrightarrow X_{i}\right)$, and by Lemma $1, X_{i} \rightsquigarrow X_{j}$.

Proof (Lemma 2). Let $X_{i}, X_{j} \subseteq A$ such that $X_{i} \rightsquigarrow X_{j}$ and $S^{\prime}$ a subset of $S$.

$(\subseteq)$ First, let us show $\Psi_{X_{i} \cup X_{j}}\left(S^{\prime}\right) \subseteq\left(\Psi_{X_{i}} \oslash \Psi_{X_{j}}\right) \circ \Omega_{X_{i} \cup X_{j}}\left(S^{\prime}\right)$. From Proposition 3.2, we know that $\Psi_{X_{i}} \circ \Psi_{X_{i} \cup X_{j}}\left(S^{\prime}\right)=\Psi_{X_{i} \cup X_{j}}\left(S^{\prime}\right)$. Thus, $\forall s \in \Psi_{X_{i} \cup X_{j}}\left(S^{\prime}\right)$, we have $[s]_{\rightleftharpoons_{X_{i}}} \subseteq \Psi_{X_{i} \cup X_{j}}\left(S^{\prime}\right)$. Similarly, an evolution by $X_{i} \cup X_{j}$ from an attractor of $X_{i}$ remain in the same attractor except potentially with evolutions by $X_{j}$. We have then, for all $s, s^{\prime} \in \Psi_{X i \cup X_{j}}\left(S^{\prime}\right)$ :

$$
\begin{aligned}
& {[s]_{\rightleftharpoons_{i}}\left[\rightarrow_{X_{i} \cup X_{j}}\right]_{\rightleftharpoons_{X_{i}}}^{*}\left[s^{\prime}\right]_{\rightleftharpoons_{i}} \Longleftrightarrow}
\end{aligned}
$$

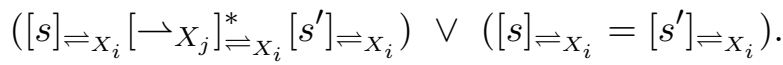

Now, since both $s$ and $s^{\prime}$ belong to attractors of $X_{i} \cup X_{j}$ (by hypothesis), if there exists an evolution by $X_{j}$ from $[s]_{\rightleftharpoons_{X_{i}}}$ to $\left[s^{\prime}\right]_{\rightleftharpoons_{X_{i}}}$, there exists obviously another path labelled by $X_{j}$ from $\left[s^{\prime}\right]_{\rightleftharpoons_{X_{i}}}$ to $[s]_{\rightleftharpoons_{X_{i}}}$. Hence, for all $s, s^{\prime} \in \Psi_{X_{i} \cup X_{j}}\left(S^{\prime}\right)$, we have:

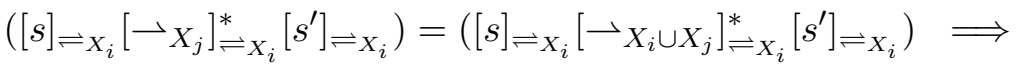

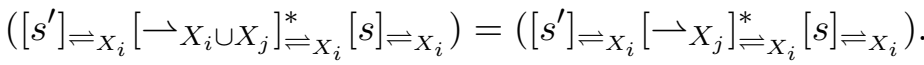

As a result, we have $[s]_{\rightleftharpoons_{X_{i}}} \in\left[\widetilde{\Psi_{X_{j}}}\right]_{\rightleftharpoons_{X_{i}}}\left(\Psi_{X_{i} \cup X_{j}}\left(S^{\prime}\right)\right)$, for all $s \in \Psi_{X_{i} \cup X_{j}}\left(S^{\prime}\right)$. Moreover, since from Proposition 2, operator $\left[\widetilde{\Psi_{X_{j}}}\right]_{\rightleftharpoons_{X_{i}}}\left(S^{\prime}\right)$ is monotone and since $\Psi_{X_{i} \cup X_{j}}\left(S^{\prime}\right) \subseteq \Omega_{X_{i} \cup X_{j}}\left(S^{\prime}\right)$, for all $s \in \Psi_{X \cup Y}\left(S^{\prime}\right)$, we can write that $[s]_{\rightleftharpoons_{X}} \in\left[\widetilde{\Psi_{X_{j}}}\right]_{\rightleftharpoons_{X_{i}}}\left(\Omega_{X_{i} \cup X_{j}}\left(S^{\prime}\right)\right)$. Now, since $s \in[s]_{一_{i}}, \forall s \in \Psi_{X_{i} \cup X_{j}}\left(S^{\prime}\right)$,

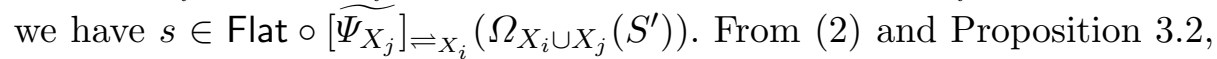


we can write:

$$
\begin{aligned}
\Psi_{X_{i}} \oslash \Psi_{X_{j}} \circ \Omega_{X_{i} \cup X_{j}}\left(S^{\prime}\right) & =\text { Flat } \circ\left[\widetilde{\Psi_{X_{j}}}\right]_{X_{i}} \circ \Psi_{X_{i}} \circ \Omega_{X_{i} \cup X_{j}}\left(S^{\prime}\right) \\
& =\text { Flat } \circ\left[\widetilde{\Psi_{X_{j}}}\right]_{\sum_{i}} \circ \Omega_{X_{i} \cup X_{j}}\left(S^{\prime}\right) .
\end{aligned}
$$

Hence, for all $s \in \Psi_{X \cup Y}\left(S^{\prime}\right)$, we have: $s \in \Psi_{X} \oslash \Psi_{Y} \circ \Omega_{X \cup Y}\left(S^{\prime}\right)$, which corresponds to the following inclusion:

$$
\Psi_{X \cup Y}\left(S^{\prime}\right) \subseteq \Psi_{X} \oslash \Psi_{Y} \circ \Omega_{X \cup Y}\left(S^{\prime}\right) .
$$

() Now, let us show $\left(\Psi_{X_{i}} \oslash \Psi_{X_{j}}\right) \circ \Omega_{X_{i} \cup X_{j}}\left(S^{\prime}\right) \subseteq \Psi_{X_{i} \cup X_{j}}\left(S^{\prime}\right)$. To do so, let us consider a state $s \in\left(\Psi_{X_{i}} \oslash \Psi_{X_{j}}\right) \circ \Omega_{X_{i} \cup X_{j}}\left(S^{\prime}\right)$. From (2) and Proposition 3.2, we have $[s]_{\rightleftharpoons_{X_{i}}} \in\left[\Psi_{X_{j}}\right]_{\rightleftharpoons_{X_{i}}} \circ\left[\Psi_{X_{i}} \circ \Omega_{X_{i} \cup X_{j}}\left(S^{\prime}\right)\right]_{\rightleftharpoons_{X_{i}}}$. Now, consider $\left[[s]_{\rightleftharpoons_{X_{i}}}\right]_{\rightleftharpoons_{X_{j}}}$. By definition of attractors, for all $s_{1}, s_{2} \in$ Flat $\circ$ Flat $\left(\left[[s]_{\rightleftharpoons_{X_{i}}}\right] \rightleftharpoons_{X_{j}}\right)$, we have $s_{1} \rightarrow_{X_{i} \cup X_{j}}^{*} s_{2}$. This means that $\left[[s]_{P_{X_{i}}}\right] \rightleftharpoons_{X_{j}}=$ $[s]_{P_{i} \cup X_{j}}$ and, as a consequence, that $s \in \Psi_{X \cup Y}\left(S^{\prime}\right)$. As a result, the inclusion $\left(\Psi_{X} \oslash \Psi_{Y}\right) \circ \Omega_{X \cup Y}\left(S^{\prime}\right) \subseteq \Psi_{X \cup Y}\left(S^{\prime}\right)$ holds.

Proof (Theorem 2). This proof is made directly by induction on the modular organisation, using Definition 6 and Lemma 2. Since $\pi=\left(X_{1}, \ldots, X_{m}\right)$ is a modular organisation, it is folding preserving and $\bigcup_{i=1}^{m-1} X_{i} \rightsquigarrow X_{m}$. Then, using (2) and Lemma 2, we have:

$$
\begin{aligned}
& \Psi_{A^{\prime}}=\left(\Psi_{\bigcup_{i=1}^{m-1} X_{i}} \oslash \Psi_{X_{m}}\right) \circ \Omega_{A^{\prime}} \\
& =\text { Flat } \circ\left[\widetilde{\Psi_{X_{m}}}\right]_{\bigodot_{i=1}^{m-1} X_{i}} \circ \Psi_{\bigcup_{i=1}^{m-1} X_{i}} \circ \Omega_{A^{\prime}} \\
& =\text { Flat } \circ\left[\widetilde{\Psi_{X_{m}}}\right]_{\bigcup_{i=1}^{m-1} X_{i}} \circ\left(\Psi_{\bigcup_{i=1}^{m-2} X_{i}} \oslash \Psi_{X_{m-1}}\right) \circ \Omega_{\bigcup_{i=1}^{m-1} X_{i}} \circ \Omega_{A^{\prime}} \\
& =\text { Flat } \circ\left[\widetilde{\Psi_{X_{m}}}\right]_{\bigcup_{i=1}^{m-1} X_{i}} \circ\left(\Psi_{\bigcup_{i=1}^{m-2} X_{i}} \oslash \Psi_{X_{m-1}}\right) \circ \Omega_{A^{\prime}} \\
& =\text { Flat } \circ\left[\widetilde{\Psi_{X_{m}}}\right] \rightleftharpoons_{\cup_{i=1}^{m-1} X_{i}} \circ\left[\widetilde{\Psi_{X_{m-1}}}\right]_{\bigcup_{i=1}^{m-2} X_{i}} \circ \Psi_{\bigcup_{i=1}^{m-2} X_{i}} \circ \Omega_{A^{\prime}} \\
& =\ldots \\
& \text { = Flat } \circ\left[\widetilde{\Psi_{X_{m}}}\right] \rightleftharpoons_{\cup_{i=1}^{m-1} X_{i}} \circ \ldots \circ\left[\widetilde{\widetilde{\Psi_{X_{2}}}}\right]_{X_{1}} \circ \Psi_{X_{1}} \circ \Omega_{A^{\prime}} .
\end{aligned}
$$

As a result, we obtain: $\Psi_{A^{\prime}}\left(S^{\prime}\right)=\left(\Psi_{X_{1}} \oslash \ldots \oslash \Psi_{X_{m}}\right) \circ \Omega_{A^{\prime}}\left(S^{\prime}\right)$, which is the expected result.

Proof (Lemma 3). The evolution is governed by the values of the regulators and the evolution concerns the states of agents of $X$ only. Under the assumptions that $X$ and $Y$ are two disjoint sets and that $R_{X} \subseteq X \cup Y$, 
the following property holds by definition of the evolution: $\forall s_{1}, s_{2} \in$ $\left.S\right|_{X \cup Y}:\left(s_{1} \rightarrow_{X} s_{2}\right) \Longleftrightarrow\left(\left.\left.\left.s_{1}\right|_{X \cup R_{X}} \rightarrow_{X} s_{2}\right|_{X \cup R_{X}} \wedge s_{1}\right|_{Y \backslash R_{X}}=\left.s_{2}\right|_{Y \backslash R_{X}}\right)$. This property extends to the transitive closure by induction:

$$
\begin{aligned}
& \forall s_{1},\left.s_{2} \in S\right|_{X \cup Y}: \\
& \quad\left(s_{1} \rightarrow_{X}^{*} s_{2}\right) \Longleftrightarrow\left(\left.\left.\left.s_{1}\right|_{X \cup R_{X}} \rightarrow_{X}^{*} s_{2}\right|_{X \cup R_{X}} \wedge s_{1}\right|_{Y \backslash R_{X}}=\left.s_{2}\right|_{Y \backslash R_{X}}\right) .
\end{aligned}
$$

First we prove that:

$$
\left.\forall s \in S\right|_{X \cup Y}:\left.s \in \Psi_{X}\left(\left.S\right|_{X \cup Y}\right) \Longleftrightarrow s\right|_{X \cup R_{X}} \in \Psi_{X}\left(\left.S\right|_{X \cup R_{X}}\right) .
$$

Let $s \in \Psi_{X}\left(\left.S\right|_{X \cup Y}\right)$. By definition of the equilibrium operator (Definition 3), $s$ complies with the following equivalent property:

$\left.\Longleftrightarrow \forall s^{\prime} \in S\right|_{X \cup Y}: s \rightarrow_{X}^{*} s^{\prime} \Longrightarrow s^{\prime} \rightarrow_{X}^{*} s$.

By application of Equation 8, we derive:

$\left.\Longleftrightarrow \forall s^{\prime} \in S\right|_{X \cup Y}:\left(\left.s\right|_{Y \backslash R_{X}}=\left.s^{\prime}\right|_{Y \backslash R_{X}}\right) \Longrightarrow$

$$
\left(\left.\left.\left.\left.s\right|_{X \cup R_{X}} \rightarrow_{X}^{*} s^{\prime}\right|_{X \cup R_{X}} \Longrightarrow s^{\prime}\right|_{X \cup R_{X}} \rightarrow_{X}^{*} s\right|_{X \cup R_{X}}\right) \text {. }
$$

Since $s \rightarrow_{X}^{*} s^{\prime}$ insures that $\left.s\right|_{X \cup R_{X}}=\left.s^{\prime}\right|_{X \cup R_{X}}$, by Equation 8, we simplify and obtain:

$\left.\Longleftrightarrow \forall s^{\prime} \in S\right|_{X \cup Y}:\left.\left.\left.\left.s\right|_{X \cup R_{X}} \rightarrow_{X}^{*} s^{\prime}\right|_{X \cup R_{X}} \Longrightarrow s^{\prime}\right|_{X \cup R_{X}} \rightarrow_{X}^{*} s\right|_{X \cup R_{X}}$. By definition of the equilibrium operator, this equivalently leads to: $\left.\Longleftrightarrow s\right|_{X \cup R_{X}} \in \Psi_{X}\left(\left.S\right|_{X \cup R_{X}}\right)$.

Now, by definition of the completion, any state $s$ complies with the following property: $\left.s \in\left(\left.s\right|_{X \cup R_{X}}\right)\right|^{Y \backslash R_{X}}$. Since $\left.s\right|_{X \cup R_{X}} \in \Psi_{X}\left(\left.S\right|_{X \cup R_{X}}\right)$, we deduce that $\left.s \in \Psi_{X}\left(\left.S\right|_{X \cup R_{X}}\right)\right|^{Y \backslash R_{X}}$. Hence, we conclude that:

$$
\left.\forall s \in S\right|_{X \cup Y}:\left.s \in \Psi_{X}\left(\left.S\right|_{X \cup Y}\right) \Longleftrightarrow s \in \Psi_{X}\left(\left.S\right|_{X \cup R_{X}}\right)\right|^{Y \backslash R_{X}} .
$$

